\title{
Comparison of ACUITY, CRUSADE, and GRACE Risk Scales for Predicting Clinical Outcomes in Patients Treated with Dual-Antiplatelet Therapy
}

\author{
Sun Young Choi ${ }^{1}$ Moo Hyun Kim ${ }^{2}$ Victor Serebruany ${ }^{3}$
}

${ }^{1}$ Department of Biomedical Laboratory Science, Daegu Health College, Daegu, Republic of Korea

2 Department of Cardiology, College of Medicine, Dong-A University, Busan, Republic of Korea

${ }^{3}$ HeartDrug ${ }^{\text {TM }}$ Research Laboratories, Johns Hopkins University,

Towson, Maryland, United States

\begin{abstract}
Address for correspondence Victor Serebruany, MD, PhD, HeartDrug ${ }^{\text {TM }}$ Research Laboratories, Johns Hopkins University, Osler Medical Building, 7600 Osler Drive, Suite 307, Towson, MD 21204 , United States (e-mail: vserebr1@jhmi.edu).
\end{abstract}

TH Open 2018;2:e399-e406.

\begin{abstract}
Keywords

- ACUITY

- CRUSADE

- GRACE

- ACS

- risk score

- bleeding

- dual-antiplatelet therapy
\end{abstract}

\section{Introduction}

Dual-antiplatelet therapy (DAPT) with aspirin and a P2 $\mathrm{Y}_{12}$ inhibitor after coronary intervention with stent implantation reduces ischemic events but increase bleeding risk, which has been associated with a critical adverse event. ${ }^{1,2}$ Numerous risk assessment scales have been introduced to better identify high-risk patients prone to bleeding and ischemic risk ${ }^{3-7}$ or vice versa. The development of a simple-to-use risk scores for bleeding and ischemia could standardize therapeutic decision making and clinical outcomes. ACUITY (Acute Catheterization and Urgent Intervention Triage Strategy) $)^{3}$ and CRUSADE (Can received

April 15, 2018 accepted after revision September 17, 2018
DOI https://doi.org/

10.1055/s-0038-1675576. ISSN 2512-9465.
๑) 2018 Georg Thieme Verlag KG
Stuttgart · New York

License terms

(c) (1) 
Rapid Risk Stratification of Unstable Angina Patients Suppress Adverse Outcomes with Early Implementation of the ACC/AHA Guidelines $)^{4}$ scores are bleeding risk algorithms. In contrast, GRACE (The Global Registry of Acute Coronary Events) ${ }^{5}$ score targeted future ischemic events and mortality. Most ischemic and bleeding risk algorithms were derived from clinical trials, and include conventional clinical and laboratory characteristics, predominantly focusing on short-term events. There are numerous reports comparing predictive values of different scales and conventional biomarkers, with somewhat mixed results. ${ }^{6,7}$ We recently show that CRUSADE score was superior to platelet testing for predicting short-term, but not 1-year, bleeding in post-percutaneous coronary intervention (PCI) Korean patients treated with DAPT. ${ }^{8}$

The aim of this study was to compare the predictive performance of CRUSADE, ACUITY, and GRACE risk scores for short-term and long-term thrombotic and bleeding events in patients treated with DAPT.

\section{Methods}

\section{Patients}

A total of 904 consecutive post-PCI patients were included (Dong-A University Medical Center, Busan, Korea). All patients received maintenance DAPT (75 mg/day clopidogrel, or $10 \mathrm{mg} /$ day prasugrel, or $180 \mathrm{mg} /$ day ticagrelor, all on top of $100 \mathrm{mg}$ aspirin), and were included in the prospective observational cross-sectional study. Written informed consent was obtained from all patients, and the study protocol was approved by the Ethical Review Board of Dong-A University Hospital. Exclusion criteria were DAPT maintenance $<1$ year, hemodynamic instability, malignancies, active bleeding or major surgery within 4 weeks, severe chronic renal failure, and treatment with other types of antiplatelet agents (e.g., cilostazol, or glycoprotein IIb/IIIa receptor blocker).

\section{Outcomes}

Major bleeding (MB) was defined according to the Bleeding Academic Research Consortium (BARC) criteria (type 3 or 5: hemodynamic instability, need for transfusion, drop in hemoglobin $\geq 3 \mathrm{~g}$, and intracranial, intraocular, or fatal bleeding). ${ }^{9}$ Major adverse cardiovascular events (MACEs) were defined as all-cause death, myocardial infarction, target vessel revascularization, and stroke. Both MB and MACE were evaluated within 1 month, and then at 12 months of followup. The ACUITY, ${ }^{3}$ CRUSADE, ${ }^{4}$ and GRACE $^{5}$ risk scores were calculated from patients' clinical characteristics available in the hospital records. ACUITY score consists of seven variables (female sex, age, type of acute coronary syndrome [ACS], unstable angina, non-ST elevation or ST elevation acute myocardial infarction, serum creatinine, and white blood cell count, all analyzed as ordinal categories). CRUSADE score was assessed with the online calculator by eight variables (female sex, diabetes mellitus, chronic heart failure, valvular heart disease, heart rate, systolic blood pressure, glomerular filtration rate, and hematocrit). GRACE score was also examined with online calculator by eight variables (age, heart rate, systolic blood pressure, serum creatinine, Killip class, cardiac arrest at admission, elevated cardiac marker, ST segment deviation). The individual rating for each variable established in each score was assigned. The total score of each patient was calculated by summing the individual result for each prognostic variable included in the score. The patients were mandatorily contacted by telephone call, or underwent personal hospital visit for outcome assessment.

\section{Statistics}

Continuous variables are expressed as means and standard deviations, while categorical variables were presented as frequencies (percentages). The comparisons between two mean values of continuous variables were analyzed using Student's $t$-test. Categorical variables were compared by using Pearson's chi-square or Fisher's exact test. The ACUITY, CRUSADE, and GRACE scores were stratified in three risk categories of bleeding as low, moderate, and high. ${ }^{3-5}$ The predictive values of ACUITY, CRUSADE, and GRACE scores were assessed by receiver operating characteristics (ROC) curve analysis (using MedCalc Version 12.2.1; MedCalc Software, Mariakerke, Belgium), applying net reclassification and integrated discrimination improvement (IDI). ${ }^{10}$ Prognostic utility of the risk models for MB and MACE has been assessed by deriving their C-statistics, using ROC curves. In general, a model with a C-statistic above 0.70 has acceptable discriminatory capacity. ${ }^{11}$ The C-statistics for the three risk models were compared with each other using a nonparametric test. $^{12}$ Net reclassification improvement (NRI) represents the average weighted improvement in discrimination. IDI considers the change in the estimated prediction probabilities as a continuous variable and represents the average improvement in predicted probability. The impact of the reclassification procedure by using the superior score was assessed by using the method of NRI. Positive values of NRI indicate a predominance of correct reclassification, while negative values indicate a predominance of incorrect reclassification. A $p$-value $<0.05$ rejects the null hypothesis of $\mathrm{NRI}=0 .{ }^{12}$ A $p$-value $<0.05$ was considered to indicate significance. Statistical analyses were performed using SPSS version 18.0 (SPSS Inc., Chicago, Illinois, United States).

\section{Results}

\section{Baseline Characteristics}

The study cohort was composed of 904 patients treated with DAPT. The baseline demographics and clinical characteristics are exhibited in - Table 1. The MB occurred in 114 patients (12.6\%) during the first 30 days, and extra 65 patients (7.2\%) at 30 days to 1 -year of follow-up. The MACE occurred in 28 (3.1\%) and $72(8.0 \%)$ patents during 30 and 30 days to 1 year, respectively. Background clinical variables and admission biomarkers were distributed differently, and depended on future MB and MACE outcomes. The future MB patients more commonly presented with non-ST-segment elevated myocardial infarction (NSTEMI) and ST-segment elevated myocardial infarction (STEMI), were older, and more frequent were females when compared with no MB patients. The MB was also associated with diabetes mellitus, hypertension, current smoking, 
Table 1 Baseline characteristics in 904 ACS patients

\begin{tabular}{|c|c|c|c|c|c|c|}
\hline Variables & MB $(n=154)$ & No MB $(n=750)$ & $p$-Value & MACE $(n=97)$ & No MACE $(n=807)$ & $p$-Value \\
\hline Age, y & $71.4 \pm 9.4$ & $64.1 \pm 10.3$ & 0.000 & $68.4 \pm 10.1$ & $65.1 \pm 10.5$ & 0.004 \\
\hline Female & $74(48.1)$ & $197(26.3)$ & 0.000 & $32(33.0)$ & $239(29.6)$ & 0.282 \\
\hline BMI, $\mathrm{kg} / \mathrm{m}^{2}$ & $23.5 \pm 3.3$ & $24.6 \pm 3.1$ & 0.000 & $23.9 \pm 2.9$ & $24.5 \pm 3.2$ & 0.107 \\
\hline Diagnosis & & & 0.000 & & & 0.083 \\
\hline Unstable angina & $70(45.5)$ & $523(69.7)$ & & $53(54.6)$ & $540(66.9)$ & \\
\hline NSTEMI & $68(44.2)$ & $193(25.7)$ & & $37(38.1)$ & $224(27.8)$ & \\
\hline STEMI & $16(10.4)$ & $34(4.5)$ & & $7(7.2)$ & $43(5.3)$ & \\
\hline Prior antiplatelet therapy & $78(50.6)$ & $394(52.5)$ & 0.368 & $71(73.2)$ & $401(49.7)$ & 0.000 \\
\hline \multicolumn{7}{|l|}{ Risk factor } \\
\hline Diabetes mellitus & $77(50.0)$ & 307 (40.9) & 0.024 & $51(52.6)$ & $333(41.3)$ & 0.022 \\
\hline Hypertension & $123(79.9)$ & $472(62.9)$ & 0.000 & $74(76.3)$ & $521(64.6)$ & 0.013 \\
\hline Dyslipidemia & 83 (53.9) & $433(57.7)$ & 0.215 & $54(55.7)$ & $462(57.2)$ & 0.424 \\
\hline Current smoking & $30(19.5)$ & $210(28.0)$ & 0.017 & $27(27.8)$ & $213(26.4)$ & 0.422 \\
\hline \multicolumn{7}{|l|}{ Past history } \\
\hline Prior MI & $36(23.4)$ & $183(24.4)$ & 0.438 & $36(37.1)$ & $183(22.7)$ & 0.002 \\
\hline Prior $\mathrm{PCl}$ & $58(37.7)$ & $311(41.5)$ & 0.217 & $59(60.8)$ & $310(38.4)$ & 0.000 \\
\hline Prior stroke & $27(17.5)$ & $66(8.8)$ & 0.002 & $18(18.6)$ & $75(9.3)$ & 0.006 \\
\hline Heart rate, bpm & $83.5 \pm 19.5$ & $73.4 \pm 13.8$ & 0.000 & $78.8 \pm 18.4$ & $74.7 \pm 15.0$ & 0.012 \\
\hline Systolic BP, mm Hg & $131.9 \pm 27.1$ & $129.7 \pm 21.7$ & 0.268 & $129.6 \pm 21.9$ & $130.1 \pm 22.9$ & 0.821 \\
\hline LVEF & $55.5 \pm 12.5$ & $59.7 \pm 10.5$ & 0.000 & $55.2 \pm 13.8$ & $59.4 \pm 10.6$ & 0.000 \\
\hline Total cholesterol, mg/dL & $166.5 \pm 48.7$ & $163.3 \pm 39.1$ & 0.404 & $152.7 \pm 42.3$ & $165.1 \pm 40.4$ & 0.007 \\
\hline WBC count $\left(10^{6} / \mathrm{mL}\right)$ & $8.8 \pm 3.8$ & $7.8 \pm 2.8$ & 0.000 & $8.2 \pm 3.0$ & $7.9 \pm 3.0$ & 0.287 \\
\hline Platelets count, $10^{3} \mu \mathrm{L}$ & $214.3 \pm 78.4$ & $210.6 \pm 58.2$ & 0.498 & $204.0 \pm 60.6$ & $212.1 \pm 62.2$ & 0.226 \\
\hline Hemoglobin, g/dL & $11.2 \pm 2.1$ & $13.1 \pm 1.7$ & 0.000 & $12.1 \pm 2.3$ & $12.9 \pm 1.9$ & 0.000 \\
\hline eGFR, mL/min, $1.73 / \mathrm{m}^{2}$ & $60.9 \pm 30.0$ & $79.2 \pm 24.1$ & 0.000 & $60.3 \pm 28.6$ & $78.0 \pm 25.1$ & 0.000 \\
\hline \multicolumn{7}{|l|}{ Bleeding risk scores } \\
\hline CRUSADE & $43.7 \pm 14.7$ & $28.4 \pm 12.6$ & 0.000 & $39.8 \pm 15.7$ & $29.9 \pm 13.7$ & 0.000 \\
\hline GRACE & $153.3 \pm 45.4$ & $118.3 \pm 32.7$ & 0.000 & $144.6 \pm 44.2$ & $121.8 \pm 35.9$ & 0.000 \\
\hline ACUITY & $18.7 \pm 6.3$ & $11.0 \pm 6.1$ & 0.000 & $16.0 \pm 7.3$ & $11.8 \pm 6.6$ & 0.000 \\
\hline
\end{tabular}

Abbreviations: BMI, body mass index; BP, blood pressure; eGFR, estimated glomerular filtration rate; MACE, major adverse cardiovascular event; MB, major bleeding; MI, myocardial infarction; LVEF, left ventricular ejection fraction; NSTEMI, non-ST-segment elevated myocardial infarction; PCI, percutaneous coronary intervention; STEMI, ST-segment elevated myocardial infarction.

previous stroke, and renal dysfunction. Similarly, MACE patients had a higher prevalence of elderly, diabetes, previous stroke, or MI, revascularization, and renal dysfunction than patients with no MACE. The mean ACUITY, CRUSADE, and GRACE scores were overall higher in patients who experienced $\mathrm{MB}$ and MACE compared with those without MB and MACE.

\section{Outcomes}

The incidence of MB and MACE is presented in - Fig. 1. The BARC type 3a defined as blood transfusion or drop in hemoglobin $\geq 3 \mathrm{~g} / \mathrm{dL}$ was observed in 98 and later in 49 patients at 30- and 30-day to 1-year follow-up, respectively. Among MACE, the most frequent outcomes were all-cause death with 13 cases for 30 days and target vessel revascularization with 34 cases for delayed 30-day to 1-year follow-up.
The frequency of MB and MACE across ACUITY, CRUSADE, and GRACE risk scores is presented in - Fig. 2. By applying previously validated criteria, 331 patients (36.6\%) based on ACUITY, 204 patients (22.6\%) based on CRUSADE, and 105 patients (11.6\%) based on GRACE scales met the threshold for the high bleeding risk category. The transition from a lower to a higher risk category carried a significant increase in $\mathrm{MB}$ and MACE rates across all risk scores.

\section{Predictive Value of Risks Scales}

- Table 2 and -Fig. 3 present the discriminatory capacity of three risk scores for MB and MACE, including assessing of the area under the curve (AUC). Applying $C$ statistics, the discriminatory ability of ACUITY (AUC: $0.82,95 \% \mathrm{CI}: 0.80-0.85$, $p<0.0001$ ) and CRUSADE (AUC: $0.82,95 \% \mathrm{CI}: 0.79-0.84$, 
e402 Predicting Outcomes during Dual-Antiplatelet Therapy Choi et al.
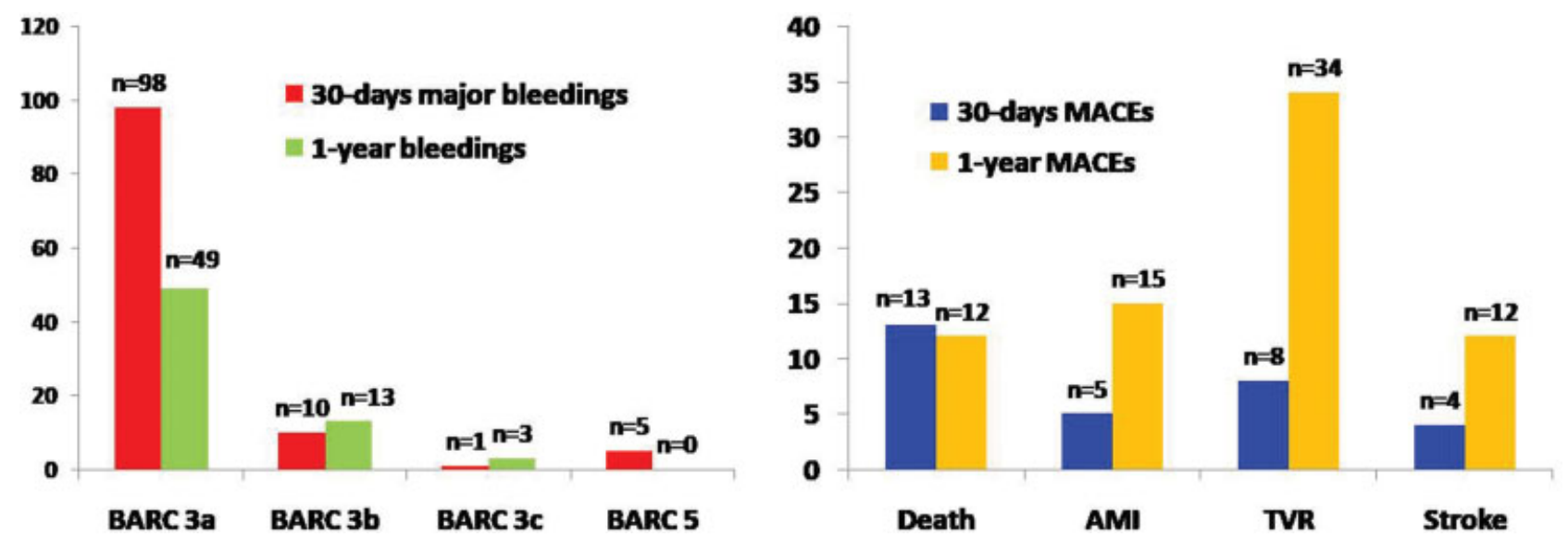

Fig. 1 Distribution of major bleeding events by BARC scale and MACE.
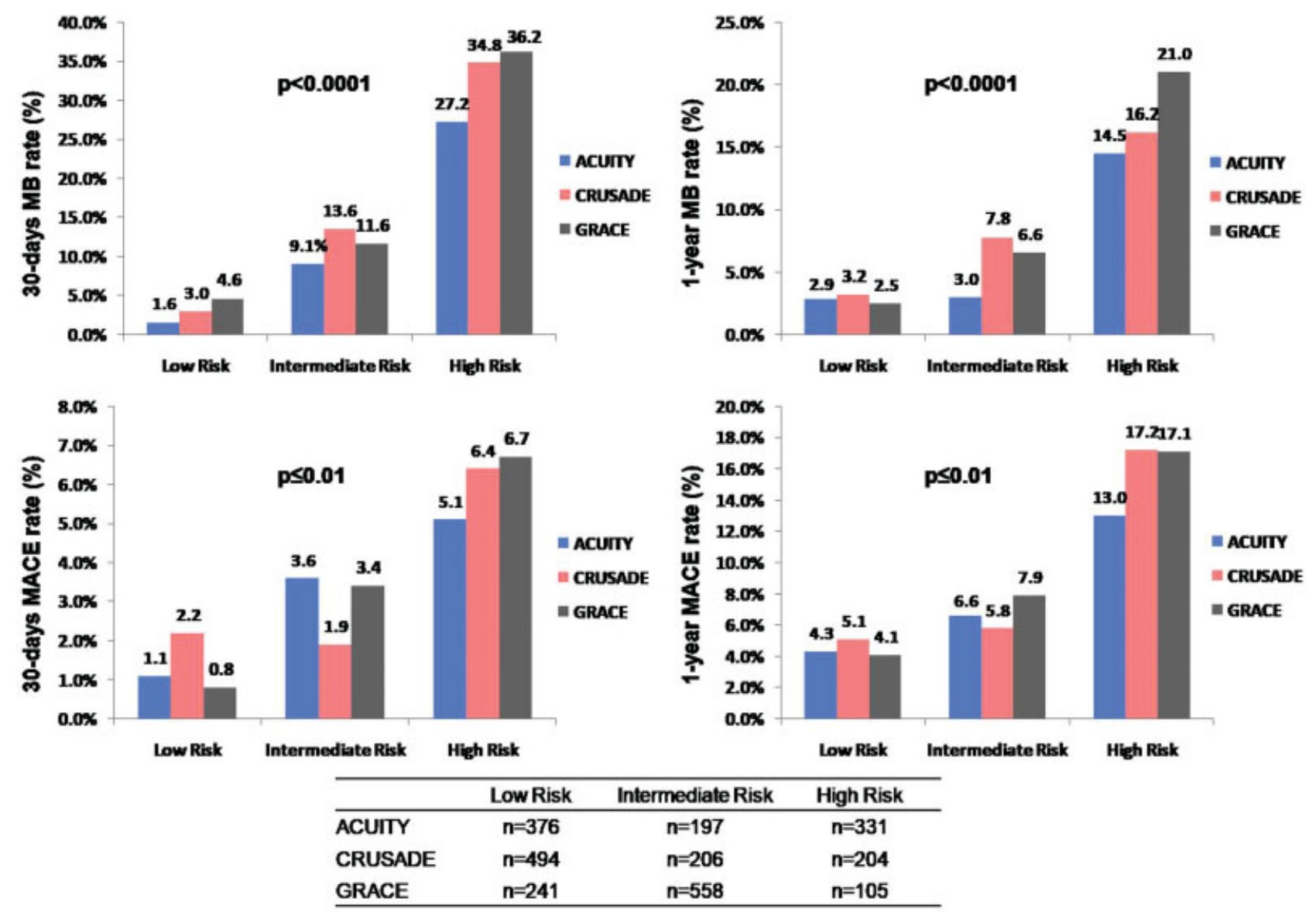

Fig. 2 Incidence of major bleeding events and MACE across ACUITY, CRUSADE, and GRACE risk score categories.

$p<0.0001$ ) for 30-day MB was similar $(p=0.76)$, but better than GRACE (AUC: $0.74,95 \% \mathrm{CI}: 0.71-0.77, p<0.0001$ ) $(p<0.001$ and $p=0.01$, respectively). These data are outlined in - Table 3. The point estimate of AUC for the prediction of 1-year MB was similar between ACUITY (AUC: 0.75 , 95\% CI: 0.72-0.77, $p<0.0001$ ), CRUSADE (AUC: $0.72,95 \% \mathrm{CI}$ : $0.69-0.75, p<0.0001$ ), and GRACE (AUC: $0.70,95 \% \mathrm{CI}: 0.67-$ $0.73, p<0.0001)$. The point estimate of AUC for the prediction of the ACUITY, CRUSADE, and GRACE risk scores for 30day and 1-year MACE, including death, was also not signifi- cantly different. ACUITY and CRUSADE risk scores successfully reclassified the risk of 30-day $M B$ compared with GRACE risk scores (-Table 4). Importantly, the CRUSADE risk score was significantly superior to GRACE or ACUITY for reclassification improvement and IDI for 1-year MACE.

\section{Discussion}

The main finding of this study is that among three conventional scores, ACUITY and CRUSADE risk scores demonstrated 
Table 2 Predictive performance of risk scores for major outcomes

\begin{tabular}{|c|c|c|c|c|}
\hline \multirow[t]{2}{*}{ Variables } & \multicolumn{2}{|l|}{ 30-d major bleeding } & \multicolumn{2}{|l|}{ 1-y major bleeding } \\
\hline & C statistics $(95 \% \mathrm{Cl})$ & $p$ & C statistics $(95 \% \mathrm{Cl})$ & $p$ \\
\hline ACUITY score & $0.83(0.81-0.86)$ & $<0.0001$ & $0.75(0.72-0.78)$ & $<0.0001$ \\
\hline CRUSADE score & $0.82(0.79-0.84)$ & $<0.0001$ & $0.73(0.70-0.76)$ & $<0.0001$ \\
\hline GRACE score & $0.74(0.71-0.77)$ & $<0.0001$ & $0.70(0.67-0.73)$ & $<0.0001$ \\
\hline \multirow[t]{2}{*}{ Variables } & \multicolumn{2}{|l|}{ 30-d MACE } & \multicolumn{2}{|l|}{ 1-y MACE } \\
\hline & C statistics $(95 \% \mathrm{Cl})$ & $p$ & C statistics $(95 \% \mathrm{Cl})$ & $p$ \\
\hline ACUITY score & $0.69(0.65-0.72)$ & 0.0003 & $0.66(0.63-0.70)$ & $<0.0001$ \\
\hline CRUSADE score & $0.64(0.61-0.67)$ & 0.0101 & $0.69(0.66-0.72)$ & $<0.0001$ \\
\hline GRACE score & $0.70(0.67-0.73)$ & 0.0001 & $0.65(0.61-0.68)$ & $<0.0001$ \\
\hline \multirow[t]{2}{*}{ Variables } & \multicolumn{2}{|l|}{ 30-d death } & \multicolumn{2}{|l|}{$1-y$ death } \\
\hline & C statistics (95\% Cl) & $p$ & C statistics $(95 \% \mathrm{Cl})$ & $p$ \\
\hline ACUITY score & $0.72(0.69-0.75)$ & 0.006 & $0.88(0.86-0.91)$ & $<0.0001$ \\
\hline CRUSADE score & $0.64(0.61-0.68)$ & 0.127 & $0.84(0.82-0.87)$ & $<0.0001$ \\
\hline GRACE score & $0.78(0.75-0.81)$ & $<0.0001$ & $0.85(0.83-0.87)$ & $<0.0001$ \\
\hline
\end{tabular}

Abbreviations: $\mathrm{Cl}$, confidence interval; MACE, major adverse cardiovascular event.

reasonably good predictive values with respect to short-term MB during DAPT when compared with GRACE. Moreover, originally designed as bleeding risk scores, they also displayed a similar capability to predict short-term and longterm mortality risks when compared with GRACE score. Importantly, the CRUSADE risk score predicted long-term MACE better than GRACE, when advanced statistics were applied. However, these data on comprehending CRUSADE and ACUITY success are somewhat mixed for two main reasons. First, patients differ substantially, somewhat neglecting that this useful score was designed exclusively for non-STEMI cohorts predicting very early in-hospital MB. Second, there are over dozen current bleeding classifications, and their inventors or/and promoters may be biased in applying their own scales at the expense of other useful algorithms. ${ }^{13,14}$ Some other integrative models, such as HASBLED, are much more simple than CRUSADE, but it is unclear how they may be implemented for the similar delayed approach to pick up either bleeding or adverse thrombotic event signal. ${ }^{15}$ Expanding original CRUSADE applicability beyond exclusive non-STEMI patients ${ }^{4}$ to the entire post-ACS pool is also important, especially considering our data in Korean patients and those facts yielded from Egyptian study yielded similar results. ${ }^{16}$ Finally, some evidence indicates the special difficulties in delayed bleeding prediction, ${ }^{17}$ what is matching well with the index dataset. That message is particularly critical since late catastrophic hemorrhages are usually the most deadly, unexpected, and hard to prevent. Our data are in full agreement with another recent study suggesting that both CRUSADE and ACUITY risk scores performed adequate discriminatory power for the prediction of MB within 30 days in ticagrelor-treated ACS patients. ${ }^{18}$ In fact, these three scores differ very significantly in our patient mixed cohort. ACUITY and GRACE include the wide scale of ACS, whereas CRUSADE applied almost exclusively to unstable angina. Furthermore, GRACE, being indicative of MACE rather than bleeding, is relatively easy to calculate and has the rather well-defined and accepted cutoff of 140 for high- (invasive) versus low-risk (conservative) patients. This consideration somewhat lowers the priority of this analyses to for clinicians. The unique meta-analyses pooling the evidence with regard to all three scales discussed here indicate their similar predictive value for bleeding risks, while accuracy of the scores increased with radial access for coronary interventions. ${ }^{19}$

\section{Strengths and Limitations}

Large sample size, reasonably validated and uniformed three established scales, and both short- and long-term observations with very careful follow-up are obvious assets. Single busy clinical center environment also reduces variability of techniques and outcomes. Each adverse event was clinically verified and confirmed. We applied the universal BARC bleeding classification, which was introduced to fairly and objectively count hemorrhages. This scale exhibits consistent growing popularity, and more frequently being validated in the major DAPT trials. Finally, we tested the abilities of three major risk scores simultaneously to discriminate outcomes, which was never done before. There are certain limitations worth mentioning. Importantly, applying a bleeding risk score to predict ischemic events (and vice versa) remains controversial. The inferior performance due to misuse of the scores is therefore not surprising. As the pathophysiology and predictors for bleeding and for MACE may differ, future studies should separately assess the comparative performance among ischemic risk scores (e.g., GRACE vs. TIMI vs. PURSUIT) and bleeding risk scores (e.g., CRUSADE vs. ACUITY). It should be 
e404 Predicting Outcomes during Dual-Antiplatelet Therapy Choi et al.
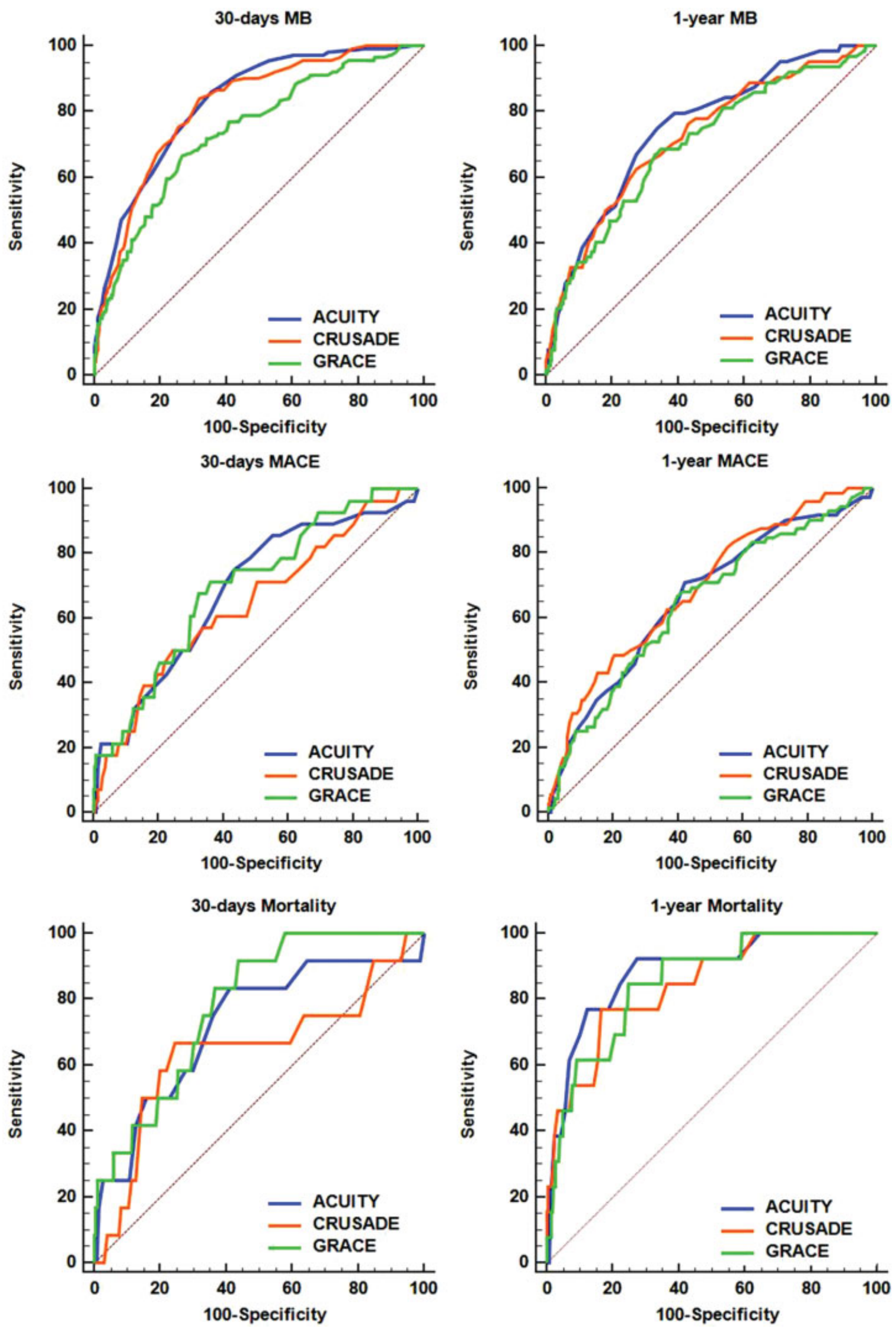

Fig. 3 Receiver operating characteristic curves for predicting major bleeding and death at 30 days and 1 year. 
Table 3 Discrimination of ACUITY versus CRUSADE versus GRACE for predicting outcomes

\begin{tabular}{|c|c|c|c|c|}
\hline \multirow[t]{2}{*}{ Variables } & \multicolumn{2}{|c|}{ 30-d major bleeding } & \multicolumn{2}{|c|}{ 1-y major bleeding } \\
\hline & z statistics & $p$ & z statistics & $p$ \\
\hline ACUITY vs. CRUSADE & 0.635 & 0.764 & 0.975 & 0.330 \\
\hline ACUITY vs. GRACE & 4.222 & $<0.0001$ & 1.624 & 0.104 \\
\hline CRUSADE vs. GRACE & 3.117 & 0.002 & 0.707 & 0.450 \\
\hline \multirow[t]{2}{*}{ Variables } & \multicolumn{2}{|c|}{ 30-days MACE } & \multicolumn{2}{|c|}{ 1-year MACE } \\
\hline & z statistics & $p$ & z statistics & $p$ \\
\hline ACUITY vs. CRUSADE & 0.895 & 0.371 & 1.030 & 0.303 \\
\hline ACUITY vs. GRACE & 0.279 & 0.780 & 0.556 & 0.578 \\
\hline CRUSADE vs. GRACE & 1.012 & 0.311 & 1.329 & 0.184 \\
\hline \multirow[t]{2}{*}{ Variables } & \multicolumn{2}{|l|}{ 30-d death } & \multicolumn{2}{|l|}{ 1-y death } \\
\hline & z statistics & $p$ & z statistics & $p$ \\
\hline ACUITY vs. CRUSADE & 1.107 & 0.268 & 0.819 & 0.413 \\
\hline ACUITY vs. GRACE & 0.978 & 0.328 & 0.643 & 0.520 \\
\hline CRUSADE vs. GRACE & 1.670 & 0.095 & 0.148 & 0.882 \\
\hline
\end{tabular}

Abbreviation: MACE, major adverse cardiovascular event.

Table 4 Risk reclassification and integrated discriminatory improvement for outcomes

\begin{tabular}{|c|c|c|c|c|c|c|c|}
\hline Comparison & Event & $\begin{array}{l}\text { Events } \\
\text { correctly } \\
\text { reclassified, } \\
\mathrm{P}(\mathrm{n} 1)\end{array}$ & $\begin{array}{l}\text { Non-events } \\
\text { correctly } \\
\text { reclassified, } \\
\mathrm{P}(\mathrm{n} 2)\end{array}$ & NRI $(95 \% \mathrm{Cl})$ & $p$ & IDI (95\% CI) & $p$ \\
\hline \multirow{6}{*}{$\begin{array}{l}\text { CRUSADE vs. } \\
\text { ACUITY }^{\mathrm{a}}\end{array}$} & 30-d MB & $0.39(44)$ & $0.39(312)$ & $-0.01(-0.19 \sim 0.17)$ & 0.929 & $0.02(0 \sim 0.05)$ & 0.070 \\
\hline & 1-y MB & $0.41(26)$ & $0.39(330)$ & $0.01(-0.22 \sim 0.25)$ & 0.918 & $-0.01(-0.02 \sim 0)$ & 0.259 \\
\hline & 30-d MACE & $0.21(6)$ & $0.26(228)$ & $-0.05(-0.41 \sim 0.32)$ & 0.811 & $0(-0.01 \sim 0.01)$ & 0.900 \\
\hline & 1-y MACE & $0.39(28)$ & $0.25(206)$ & $0.14(-0.08 \sim 0.36)$ & 0.250 & $0.01(0 \sim 0.02)$ & 0.031 \\
\hline & 30-d death & $0.5(6)$ & $0.37(328)$ & $0.13(-0.36 \sim 0.63)$ & 0.649 & $0(-0.01 \sim 0.01)$ & 0.736 \\
\hline & 1 -y death & $0.69(9)$ & $0.63(561)$ & $0.06(-0.33 \sim 0.46)$ & 0.822 & $0.01(0 \sim 0.01)$ & 0.273 \\
\hline \multirow{6}{*}{$\begin{array}{l}\text { ACUITY vs. } \\
\text { GRACE }^{\mathrm{a}}\end{array}$} & 30-d MB & $0.11(12)$ & $0.43(342)$ & $-0.54(-0.73 \sim-0.35)$ & 0.000 & $-0.05(\mathrm{v} 0.07 \sim-0.02)$ & 0.000 \\
\hline & 1-y MB & $0.03(2)$ & $0.07(62)$ & $-0.11(-0.36 \sim 0.15)$ & 0.418 & $0.01(-0.01 \sim 0.02)$ & 0.548 \\
\hline & 30-d MACE & $0.29(8)$ & $0.38(330)$ & $-0.09(-0.45 \sim 0.27)$ & 0.636 & $0(0 \sim 0.01)$ & 0.684 \\
\hline & 1-y MACE & $0.03(2)$ & $0.07(58)$ & $-0.04(-0.28 \sim 0.2)$ & 0.733 & $0(-0.01 \sim 0.01)$ & 0.695 \\
\hline & 30-d death & $0.17(2)$ & $0.07(62)$ & $0.24(-0.33 \sim 0.8)$ & 0.416 & $0(-0.01 \sim 0.01)$ & 0.871 \\
\hline & 1-y death & $0.23(3)$ & $0.07(63)$ & $0.3(-0.23 \sim 0.83)$ & 0.281 & $0.02(0 \sim 0.04)$ & 0.103 \\
\hline \multirow{6}{*}{$\begin{array}{l}\text { CRUSADE vs. } \\
\text { GRACE }^{\mathrm{a}}\end{array}$} & 30-d MB & $0.14(16)$ & $0.37(290)$ & $-0.51(-0.7 \sim-0.31)$ & 0.000 & $-0.07(-0.1 \sim-0.04)$ & 0.000 \\
\hline & 1-y MB & $0(0)$ & $0.15(122)$ & $-0.15(-0.4 \sim 0.11)$ & 0.263 & $0(-0.02 \sim 0.02)$ & 0.933 \\
\hline & 30-d MACE & $0.35(10)$ & $0.19(164)$ & $0.17(-0.18 \sim 0.52)$ & 0.376 & $0(-0.01 \sim 0.01)$ & 0.838 \\
\hline & 1-y MACE & $0.22(16)$ & $0.6(48)$ & $-0.28(-0.52 \sim-0.04)$ & 0.023 & $-0.02(-0.03 \sim 0)$ & 0.034 \\
\hline & 30-d death & $0.33(4)$ & $0.19(170)$ & $0.14(-0.39 \sim 0.68)$ & 0.623 & $0(-0.01 \sim 0.01)$ & 0.848 \\
\hline & 1-y death & $0.54(7)$ & $0.19(167)$ & $0.35(-0.11 \sim 0.81)$ & 0.209 & $0.01(-0.01 \sim 0.03)$ & 0.206 \\
\hline
\end{tabular}

Abbreviations: MACE, major adverse cardiovascular event; MB, major bleeding.

${ }^{a}$ The model considered each bleeding risk score as a reference value for the others.

emphasized that there might be important confounders to our analysis potentially impacting the conclusions including potentially missed outcomes. Among most important limitations are nonrandomized observational cross-sectional design, background differences among the patients, and pooled analyses of various stenting techniques (the index dataset). There were very few ( $10 \%$ ) classical STEMI patients, limiting extrapolation of the index data to more "heavy" ischemic cohorts. We used a "real-life" registry, acknowledging that minority of prasugrel and ticagrelor patients may 
compromise the homogeneity of clopidogrel data, potentially increasing the statistical "noise." Another shortcoming is the fact that we did not capture minor bleeding events, limiting the clinical applicability of the index dataset. Future studies should definitely focus more on minor hemorrhagic complications, which are critical for compliance, and drug discontinuations. In this study, we deliberately focus on delayed bleeding risks, also acknowledging that most bleeds occur early, and those were missed since we used up to 12 months' lag in capturing events. From a pragmatic point of view, considering recently discovered association of malignancies, potency of antiplatelet agents, and bleeding, ${ }^{20}$ we now feel that it was a mistake to exclude cancer patients from this registry. It would be also useful to count minor bleeding events, which has not been done here. With respect to DAPT and especially for bleeding risk prediction, it would be interesting in the future to match how the real "DAPT risk scores" perform here. It will be important to include PRECISE-DAPT, DAPT, TIMI score, and/ or PARIS score to the further analysis. ${ }^{21}$ The first two specifically could be important as they are included in the recent ESC DAPT 2017 guidelines. Also, as with any conventional antiplatelet studies, with very few exceptions, compliance with drugs was not assessed. Finally, our study was conducted exclusively in Korean cohort; so, extrapolation of these facts into other ethnicities seems premature.

In conclusion, ACUITY and CRUSADE scores were superior to GRACE in predicting 30-day bleeding. However, all three risk scales were similarly reliable for long-term 1-year hemorrhages, and thrombotic events assessment in Korean patients. Further evidence should be urgently retrieved from large unbiased uniformed government national registries or large well-controlled insurance claims datasets.

\section{Funding}

This research was supported by a grant from the Korea Health Technology R\&D Project through the Korea Health Industry Development Institute (KHIDI), funded by the Ministry of Health \& Welfare, Republic of Korea (grant number: HI14C1731) and funded by the Ministry of Education, Science and Technology (NNRF-2015R1D1A1A09057 025) to M.H.K. Part of this work was supported by the "Brain Pool" program funded by the Korean Ministry of Science and Technology to V.S.

\section{Conflict of Interest}

None declared.

\section{References}

1 Montalescot G, Brieger D, Dalby AJ, Park SJ, Mehran R. Duration of dual antiplatelet therapy after coronary stenting: a review of the evidence. J Am Coll Cardiol 2015;66(07):832-847

2 Giustino G, Baber U, Sartori S, et al. Duration of dual antiplatelet therapy after drug-eluting stent implantation: a systematic review and meta-analysis of randomized controlled trials. J Am Coll Cardiol 2015;65(13):1298-1310

3 Mehran R, Pocock SJ, Nikolsky E, et al. A risk score to predict bleeding in patients with acute coronary syndromes. J Am Coll Cardiol 2010;55(23):2556-2566
4 Subherwal S, Bach RG, Chen AY, et al. Baseline risk of major bleeding in non-ST-segment-elevation myocardial infarction: the CRUSADE (Can Rapid risk stratification of Unstable angina patients Suppress ADverse outcomes with Early implementation of the ACC/AHA Guidelines) Bleeding Score. Circulation 2009;119(14):1873-1882

5 Granger CB, Goldberg RJ, Dabbous O, et al; Global Registry of Acute Coronary Events Investigators. Predictors of hospital mortality in the global registry of acute coronary events. Arch Intern Med 2003;163(19):2345-2353

6 Costa F, Tijssen JG, Ariotti S, et al. Incremental value of the CRUSADE, ACUITY, and HAS-BLED risk scores for the prediction of hemorrhagic events after coronary stent implantation in patients undergoing long or short duration of dual antiplatelet therapy. J Am Heart Assoc 2015;4(12):e002524

7 Correia LC, Garcia G, Kalil F, et al. Prognostic value of TIMI score versus GRACE score in ST-segment elevation myocardial infarction. Arq Bras Cardiol 2014;103(02):98-106

8 Bang J, Choi SY, Kim MH, Serebruany V. CRUSADE score is superior to platelet function testing for prediction of bleeding in patients following coronary interventions. EBioMedicine 2017;21:213-217

9 Mehran R, Rao SV, Bhatt DL, et al. Standardized bleeding definitions for cardiovascular clinical trials: a consensus report from the Bleeding Academic Research Consortium. Circulation 2011;123 (23):2736-2747

10 Pencina MJ, D’Agostino RB Sr, D’Agostino RB Jr, Vasan RS. Evaluating the added predictive ability of a new marker: from area under the ROC curve to reclassification and beyond. Stat Med 2008;27 (02):157-172, discussion 207-212

11 Núñez E, Steyerberg EW, Núñez J. [Regression modeling strategies]. Rev Esp Cardiol 2011;64(06):501-507

12 DeLong ER, DeLong DM, Clarke-Pearson DL. Comparing the areas under two or more correlated receiver operating characteristic curves: a nonparametric approach. Biometrics 1988;44(03):837-845

13 Biancari F, Brascia D, Onorati F, et al. Prediction of severe bleeding after coronary surgery: the WILL-BLEED Risk Score. Thromb Haemost 2017;117(03):445-456

14 Steg PG, Huber K, Andreotti F, et al. Bleeding in acute coronary syndromes and percutaneous coronary interventions: position paper by the Working Group on Thrombosis of the European Society of Cardiology. Eur Heart J 2011;32(15):1854-1864

15 Hsieh MJ, Lee CH, Chen CC, Chang SH, Wang CY, Hsieh IC. Predictive performance of HAS-BLED risk score for long-term survival in patients with non-ST elevated myocardial infarction without atrial fibrillation. J Cardiol 2017;69(01):136-143

16 Al-Daydamony MM, Farag EM. CRUSADE bleeding score as a predictor of bleeding events in patients with acute coronary syndrome in Zagazig University Hospital. Indian Heart J 2016; 68(05):632-638

17 Garay A, Ariza-Solé A, Abu-Assi E, Lorente V, Sánchez-Salado JC, Cequier Á. Mid term bleeding risk prediction after an acute coronary syndrome: an unsolved question. Rev Esp Cardiol (Engl Ed) 2016;69 (05):527-529

$18 \mathrm{Xi} \mathrm{S}$, Zhou S, Wang X, et al. The performance of CRUSADE and ACUITY bleeding risk scores in ticagrelor-treated ACS patients who underwent PCI. Thromb Haemost 2017;117(11): 2186-2193

19 Taha S, D'Ascenzo F, Moretti C, et al. Accuracy of bleeding scores for patients presenting with myocardial infarction: a meta-analysis of 9 studies and 13759 patients. Postepy Kardiol Interwencyjnej 2015;11(03):182-190

20 Food and Drug Administration. Clinical Review on Cancer Risk. Available at: http://www.accessdata.fda.gov/drugsatfda_docs/nda/ 2015/2063160rig1Orig2s000 MedR.pdf. Assessed April 12, 2018

21 Amador P, Santos JF, Gonçalves S, Seixo F, Soares L. Comparison of ischemic and bleeding risk scores in non-ST elevation acute coronary syndromes. Acute Card Care 2011;13(02):68-75 